\title{
Biological determinants of physical activity across the life course: a "Determinants of Diet and Physical Activity" (DEDIPAC) umbrella systematic literature review
}

Katina Aleksovska ${ }^{1 \dagger}$, Anna Puggina ${ }^{1 *+}$, Luca Giraldi ${ }^{1}$, Christoph Buck ${ }^{2}$, Con Burns ${ }^{3}$, Greet Cardon ${ }^{4}$, Angela Carlin ${ }^{5}$, Simon Chantal ${ }^{6}$, Donatella Ciarapica ${ }^{7}$, Marco Colotto ${ }^{1}$, Giancarlo Condello ${ }^{8}$, Tara Coppinger $^{3}$, Cristina Cortis ${ }^{9}$, Sara D'Haese ${ }^{4}$, Marieke De Craemer ${ }^{4}$, Andrea Di Blasio ${ }^{10}$, Sylvia Hansen ${ }^{11}$, Licia lacoviello ${ }^{12}$, Johann Issartel ${ }^{13}$, Pascal Izzicupo ${ }^{10}$, Lina Jaeschke ${ }^{14}$, Martina Kanning ${ }^{11}$, Aileen Kennedy ${ }^{15}$, Fiona Ling ${ }^{5,16}$, Agnes Luzak ${ }^{17}$, Giorgio Napolitano ${ }^{10}$, Julie-Anne Nazare ${ }^{18}$, Camille Perchoux ${ }^{18}$, Tobias Pischon ${ }^{14}$, Angela Polito ${ }^{7}$, Alessandra Sannella ${ }^{9}$, Holger Schulz ${ }^{17}$, Rhoda Sohun ${ }^{5}$, Astrid Steinbrecher ${ }^{14}$, Wolfgang Schlicht ${ }^{11}$, Walter Ricciardi ${ }^{1,19}$, Ciaran MacDonncha ${ }^{5 \dagger}$, Laura Capranica ${ }^{8 \dagger}$ and Stefania Boccia ${ }^{1,20 \dagger}$

\begin{abstract}
Background: Despite the large number of studies and reviews available, the evidence regarding the biological determinants of physical activity (PA) is inconclusive. In this umbrella review, we summarized the current evidence on the biological determinants of PA across the life course, by pooling the results of the available systematic literature reviews (SLRs) and meta-analyses (MAs).

Methods: We conducted an online search on MEDLINE, ISI Web of Science, Scopus, and SPORTDiscus databases up to January 2018. SLRs and MAs of observational studies that investigated the association between biological determinants of PA and having PA as outcome were considered eligible. The extracted data were assessed based on the importance of the determinants, the strength of evidence, and the methodological quality.
\end{abstract}

Results: We identified 19 reviews of which most were of moderate methodological quality. Determinants that were studied most frequently among all ages and demonstrated evidence suggesting a positive association to PA were younger age, being male, higher health status, and higher physical fitness levels. Among adults, normal birth weight was found to be positively associated to PA with convincing strength of evidence, while findings among adolescents were inconsistent and with limited strength of evidence.

Conclusions: Different social or behavioral factors may contribute to the decrease of PA with age and among females versus males, and creating programmes targeted at diverse ages, female population, and adults with abnormal birth weight is recommended. Future studies should use prospective study designs, standardized definitions of PA, and objective measurement methods of PA assessment.

Keywords: Physical activity, Biological determinants, Umbrella systematic review

\footnotetext{
* Correspondence: annapuggina@gmail.com

† Katina Aleksovska, Anna Puggina, Ciaran MacDonncha, Laura Capranica and

Stefania Boccia contributed equally to this work.

${ }^{1}$ Università Cattolica del Sacro Cuore, Sezione di Igiene, Istituto di Sanità

Pubblica, Rome, Italy

Full list of author information is available at the end of the article
} 


\section{Key Points}

- Younger age, being male, higher health status and higher physical fitness levels suggested a positive association with physical activity.

- Normal birth weight was positively associated with physical activity among adults.

- Different social and behavioral factors contribute to the decrease of physical activity with increasing age.

\section{Background}

The World Health Organization (WHO) has developed global recommendations to increase the amount of physical activity (PA) in the general population, following the abundant evidence of the positive effects of PA on the maintenance of cardiovascular health and metabolic index, thus being of high importance for the prevention and the management of the non-communicable diseases (NCDs) [1]. Since NCDs constitute a large part of the worldwide disease burden, prevention programs with the effective incorporation of PA are of paramount importance [1-3].

Biological determinants can be all the individual characteristics of a person that have biological background, including genetics, family predisposition, pathology, health status, anthropometry, body mass index (BMI)/adiposity, birth weight, physical fitness levels, age, sex, ethnicity, etc. [4]. Even though some of them are non-modifiable, they influence the patterns of PA interacting with other factors on multiple levels [5-8]. Because of that, they should be considered when investigating PA participation and introducing new interventions of PA.

Several original studies, systematic literature reviews (SLRs), and meta-analyses (MAs) evaluating the determinants promoting or inhibiting PA participation are available in the literature. Specifically concerning biological determinants of PA, a number of primary epidemiological studies, SLRs and MAs, and two umbrella reviews $[9,10]$, the last concerning only young populations, have been published. According to all these studies, lower age and being male were generally found to be positively associated with PA in most of them and there is inconsistent evidence for the association between PA and several additional biological determinants (e.g., BMI, ethnicity, health status, and family risk). Among studies, there is wide variability of study aims and measurement methods and classifications used in assessing PA. This produces variability of study results and as a result, a lack of precise evidence about the biological determinants of PA participation. Furthermore, in order to establish experimental evidence related to PA, a clear understanding of associations or predictive relationships between determinants is needed [11].
Hence, the aim of this umbrella systematic review is to give an overview of the studies investigating biological determinants influencing PA across the life course by systematically reviewing the available evidence from existing SLRs and MAs (referred as "reviews" in the text) of primary observational studies. As PA is beneficial for health of people of any age, we did not restrict the overview to a particular age group. Additionally, we assessed the overall results of the retrieved reviews in terms of the importance of the determinant, the strength of the evidence, and the methodological quality of the reviews.

\section{Methods}

This umbrella review is part of the "Determinants of Diet and Physical Activity" (DEDIPAC) project (https:// www.dedipac.eu/), which was planned to include seven umbrella reviews on determinants of PA (biological, psychological, behavioral, physical, socio-cultural, economic, and policy). The current umbrella review focuses solely on the biological determinants of PA.

We drafted this manuscript following the PRISMA checklist [12]. The protocol of the umbrella systematic review is registered on PROSPERO (Record ID: CRD42 015010616), the international prospective register of systematic reviews [13].

\section{Search Strategy and Eligibility Criteria}

We used the same search strategy for all the seven umbrella reviews, extracting at the end only the articles that included biological determinants. We systematically searched electronic databases for SLRs and MAs investigating the determinants of PA across the life course. An online search was conducted on the following search engines: MEDLINE, ISI Web of Science, Scopus, and SPORTDiscus. The search was limited to reviews published in English language from January 2004 to January 2018. In order to summarize the current knowledge on determinants of PA, we did not include the reviews published before 2004. Table 1 shows the MEDLINE search strategy; this was also used as the template for the search strategies in the other databases.

SLRs and MAs of observational primary studies, done on participants at any age, on the association between any determinant and PA, or exercise, or sport as main outcome, were included in the umbrella review. The following were excluded: (i) SLRs and MAs of intervention studies; (ii) SLRs and MAs that did not focus on the general population (e.g., reviews of studies done on patients, athletes, specific professions); and (iii) umbrella systematic reviews on the same topic (e.g., reviews of SLRs or MAs of epidemiological studies on determinants associated with PA). 
Table 1 Search strategy: key words used for the literature research

\begin{tabular}{l} 
Set Search terms \\
\hline$\# 1 \quad$ "physical activit*" OR "physical exercise*" OR sport OR "motor activit*" OR "locomotor activit*" OR athletic* OR fitness OR "physical movement*" \\
OR "physical performance*" OR "aerobic exercise*" OR "physical effort*" OR "physical exertion*" \\
\#2 determinant OR determinants OR correlator OR correlators OR mediator OR mediators OR moderator OR moderators OR contributor OR \\
contributors OR factor OR factors OR association OR modifier OR modifiers OR confounder OR confounders OR pattern OR patterns OR \\
predictor* \\
\#3 demographic* OR motivation OR cognition OR emotion* OR attitude* OR "self-perception" OR "self-confidence" OR "self-efficacy" OR \\
competence OR reward* OR success* OR challenge* OR knowledge OR belief* OR "personal trait*" OR "body image" OR satisfaction OR "time \\
availability" OR "perceived environment" OR family OR peer* OR school* OR leader* OR coach* OR group* OR "climate" OR network* OR \\
employment OR retirement OR "educational level" OR SES OR "socioeconomic status" OR "local identity" OR "national identity" OR value* OR \\
tradition* OR "social expectation*" OR "social trend*" OR "social barriere*" OR "availability of tool*" OR "availability of service*" OR "access to tool*" \\
OR "access to service*" OR neighborhood OR "community route*" OR "school environment" OR "work environment" OR architecture OR \\
urbanization OR transport OR traffic OR "facilit* in public space*" OR advertisement OR "availability of sport club*" OR "availability of fitness \\
center*" OR advocacy OR lobbying OR "corporate social responsibility" OR "physical activity promotion initiativ*" OR legislation OR health OR \\
education OR tourism OR environment OR "urban planning" OR transport* OR sport OR sports OR culture OR dance OR theater OR "gender \\
mainstreaming" OR "social inclusion" OR "fiscal measure*" OR program* OR plan OR plans OR communication OR media OR guideline* \\
"systematic literature review" OR "meta-analysis"
\end{tabular}

\section{Selection Process}

Across all databases, our search identified a total number of 18,516 potentially relevant papers. After the removal of duplicates, 15,147 papers remained. Relevant papers were independently screened and assessed by two reviewers belonging to the DEDIPAC $\mathrm{KH}$ (Knowledge $\mathrm{Hub}$ ), who screened the titles and if necessary, the abstracts, and the full texts. Before the final study inclusion or exclusion, a common decision was reached for each study. Any uncertainty and disagreement was resolved by consulting three further authors (SB, LC, AP).

As summarized in Fig. 1, after title and abstract reading, 12,414 and 2198 articles were respectively excluded because they did not meet the inclusion criteria. Thus, a total number of 535 full-text articles were assessed for eligibility, which resulted in inclusion of 63 eligible papers. Of these, 44 reviews did not concern biological determinants of PA. Therefore, the final number of reviews included in the present umbrella review on biological determinants of PA was 19.

\section{Data Extraction}

For each included review, we extracted data on predefined extraction forms, developed by the two authors (KA, AP) and verified by the DEDIPAC $\mathrm{KH}$, which include the following information: year of publication, type of review (SLR or MA), number of eligible primary studies included over the total number of studies included in each review; continent/s of the included studies, primary study design, overall sample size, age range or mean age, sex proportion, year of publication range of included studies, outcome details, type of

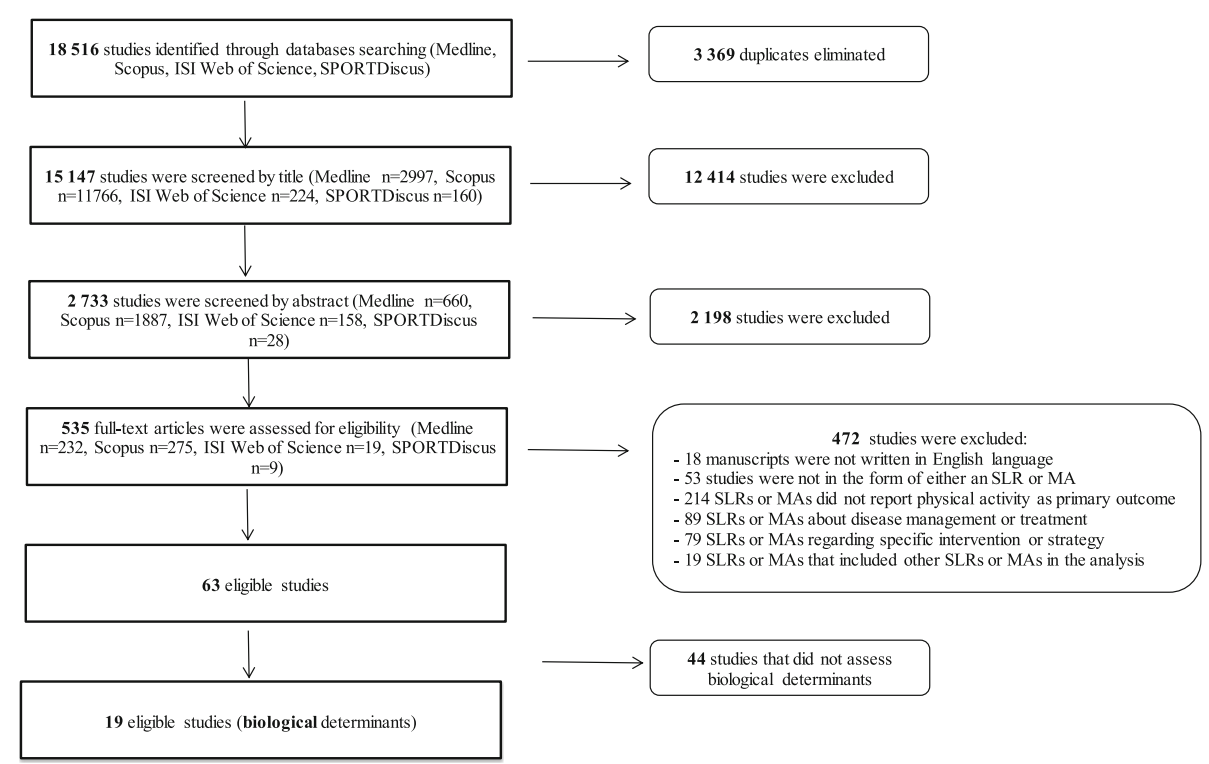

Fig. 1 Flowchart of the literature research by database. MA meta-analysis, SLR systematic literature review 
determinant/s, aim of the review; overall results (qualitative or quantitative), overall recommendations, and limitations as provided by the review itself.

\section{Evaluation of Importance of Determinants and Strength of the Evidence}

We summarized the retrieved results from the eligible studies combining two grading scales, used previously by Sleddens et al. [14]. One of the scales grades the importance of the determinants (referring to the consistency of the associations among reviews/individual studies) and the other grades the strength of evidence (referring to the study design used among individual studies).

According to the scale for the importance, a determinant can score a (--) if all reviews, without exception report no association between the determinant and the outcome, a (-) if the association was found in less than $25 \%$ of the reviews or of the original studies, and a (0) if the results are mixed, or more specifically, that the variable has been found to be a determinant and/or reported a (non)-significant effect size larger than 0.30 in $25 \%$ to $75 \%$ of the available reviews or of the primary studies analyzed in these reviews. Furthermore, the importance of the determinant scores a $(+)$ if the association was found in more than $75 \%$ of the reviews or of the included individual studies and a $(++)$ if association was found in all reviews, without exception.

The strength of the evidence is described as "convincing" (convincing evidence, Ce) if it is based on studies that show consistent associations and have longitudinal design with sufficient size and duration, whereas evidence of "probable" association (probable evidence, Pe) can be given to determinants showing fairly consistent associations based upon at least two cohort studies. In the second case, there are some shortcomings either in terms of the consistency of the results or other aspects such as limited duration of the studies, small sample sizes, or inadequate follow up. Furthermore, "limited suggestive evidence" (Ls) is given to determinants for which there is insufficient number of longitudinal studies and "limited, no conclusive evidence" (Lns) when the evidence for the associations between a determinant and the outcome are based solely on studies of cross-sectional design [14].

\section{Quality Assessment}

We assessed the methodological quality of the included reviews using a modified version of the AMSTAR Checklist [15]. The question number 11 referring to the presence of any conflict of interest was modified after a consensus between the DEDIPAC KH partners, so that the conflict of interest was evaluated in the reviews included and not in the primary studies included in each review.
The included SLRs and MAs were independently evaluated by two reviewers belonging to the DEDIPAC KH. Any uncertainty and disagreement was resolved by consulting three further authors (SB, LC, AP). The eleven criteria were evaluated and scored as a 1 when the criterion was applicable to the analyzed review or as a 0 when the criterion was not applicable to the analyzed review. As a consequence, the total quality score for each included review ranged from 0 to 11 . The quality of the review was labeled as weak (score ranging from 0 to 3 ), moderate (score ranging from 4 to 7 ), or strong (score ranging from 8 to 11$)$.

\section{Results}

\section{Characteristics of the SLRs and MAs Included}

The characteristics of the 19 included SLRs and MAs (14 and 5 respectively) are summarized in Table 2. Since some of them included primary studies that examined the associations between non-biological determinants and PA, we did not appraise all the primary studies included in the individual SLRs or MAs in our umbrella review.

Most of the reviews included primary studies from multiple continents, mostly Europe (14 reviews), North America (13 reviews), and Australia (8 reviews). One review included cohort studies conducted only in Europe [16]. In 11 of the included reviews, most of the primary studies were cross-sectional [16-27], but there was also a considerable number of reviews that included prospective and cohort studies $[16,18,20-22,24,26,28-$ 31]. In six reviews, it was not possible to retrieve the total population sample size of the included studies [17$20,32,33$, and two reviews provided only the sum of the individual studies' sample sizes [23, 29]. In the remaining studies, the total population sample size ranged from 878 to 522,967 . Some reviews did not report the age of the participants in the primary studies $[24,33]$. Finally, the percentage of the female participants, if reported, ranged from 0 to $100 \%$ of the total sample size, though these data were absent in some studies [17-20, 32-34] (Table 2).

\section{Investigated Determinants of the Reviews}

Table 3 summarizes the findings of the included reviews on the associations between the biological determinants of PA. The most frequently studied determinants were age $(n=13)[17-23,25,26,28,29,32,34]$, sex $(n=14)$ $[17-21,23-26,28,29,32-34]$, and ethnicity $(n=10)$ [17-21, 24, 28, 29, 32, 33]. BMI or overweight were assessed in nine reviews [17, 19-22, 25, 28, 32, 34]; two reviews included the family risk in their investigations $[18,21]$, five reviews examined the health status of the participants [21, 22, 25, 27, 32], and six reviews investigated physical fitness levels/motor function/motor skills/ 


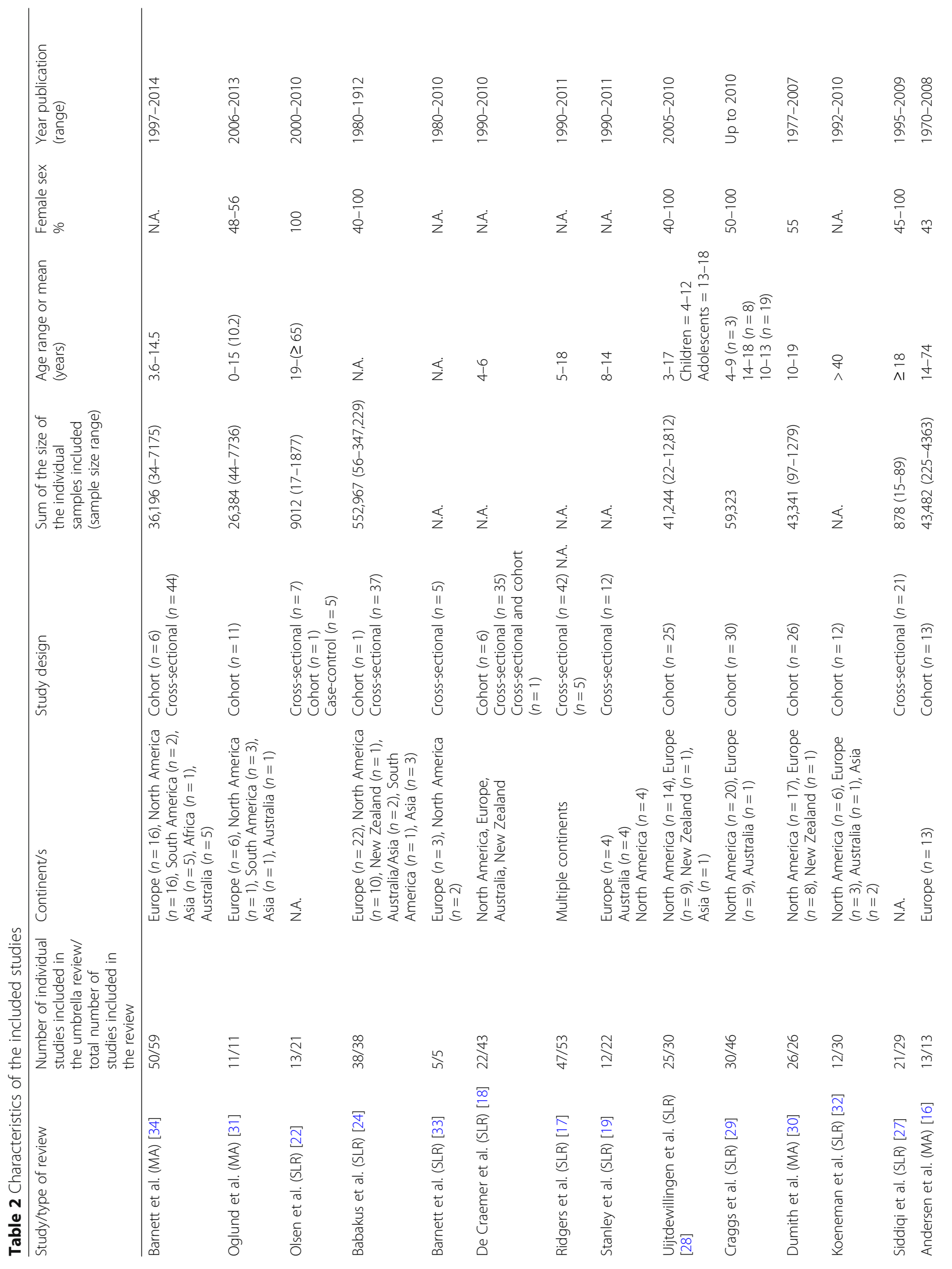




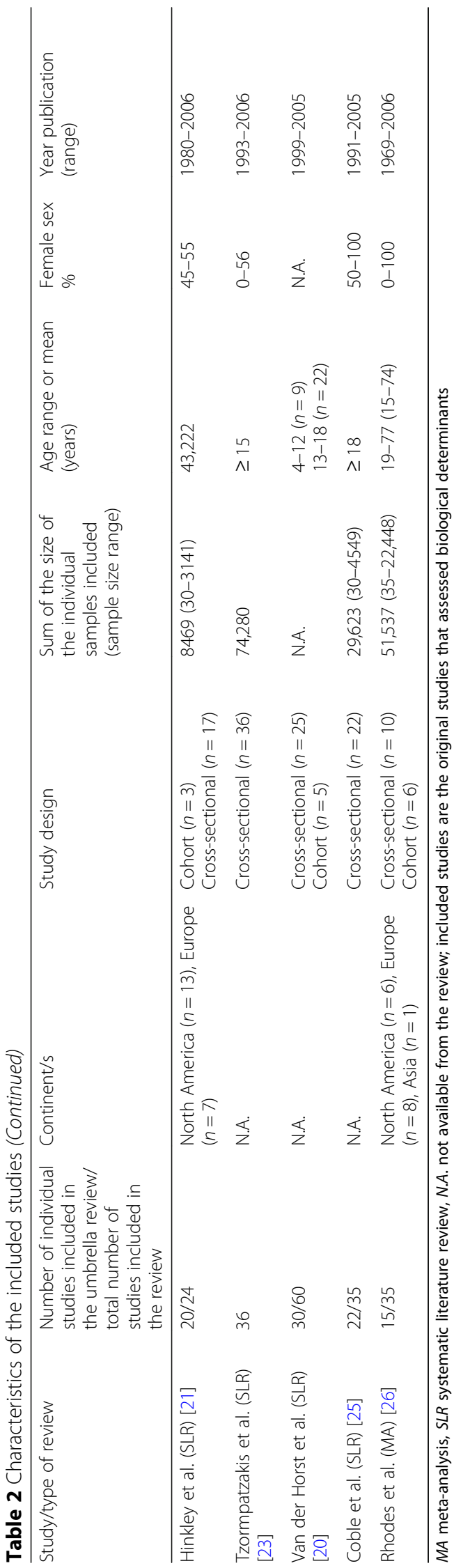




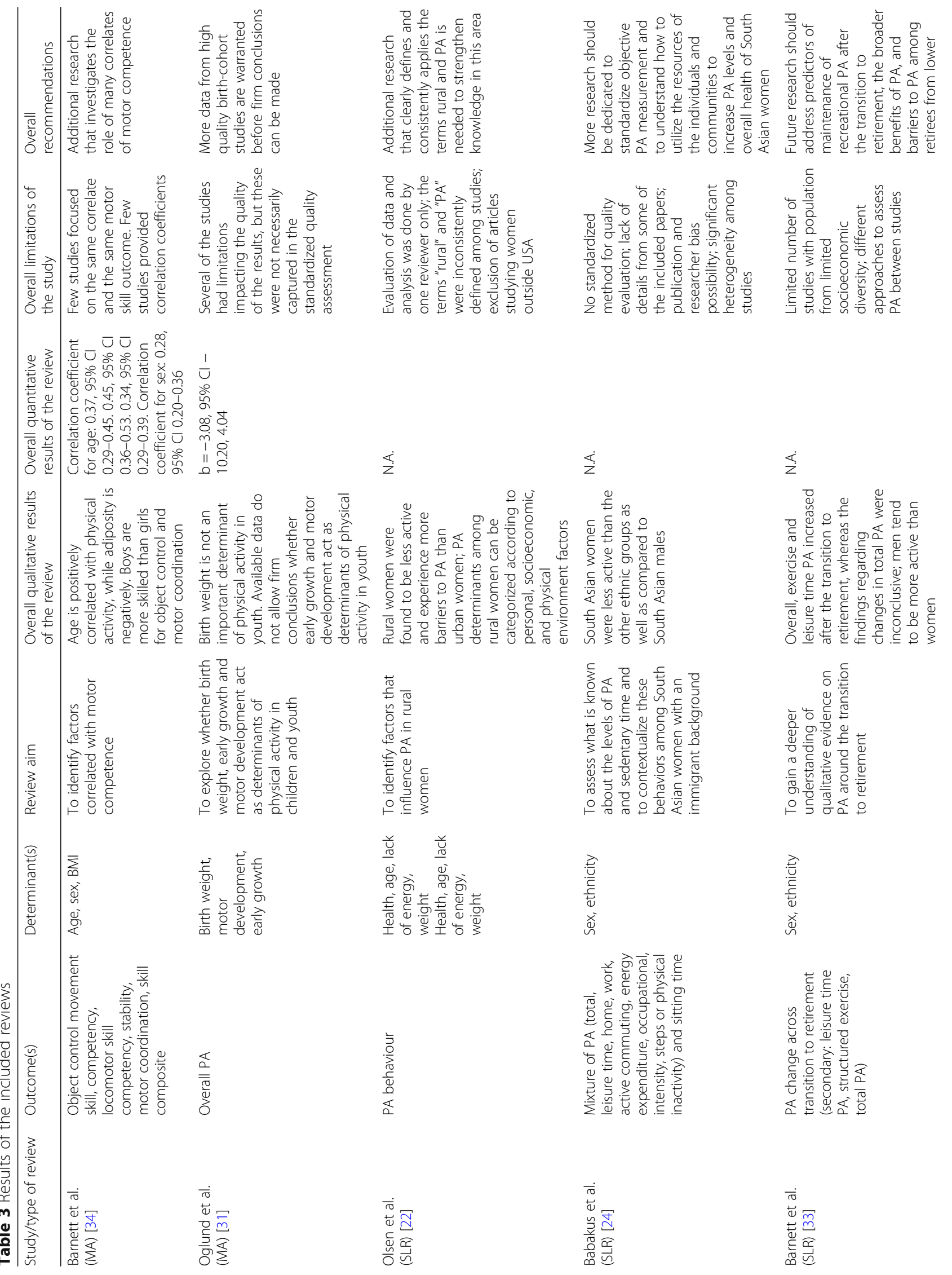




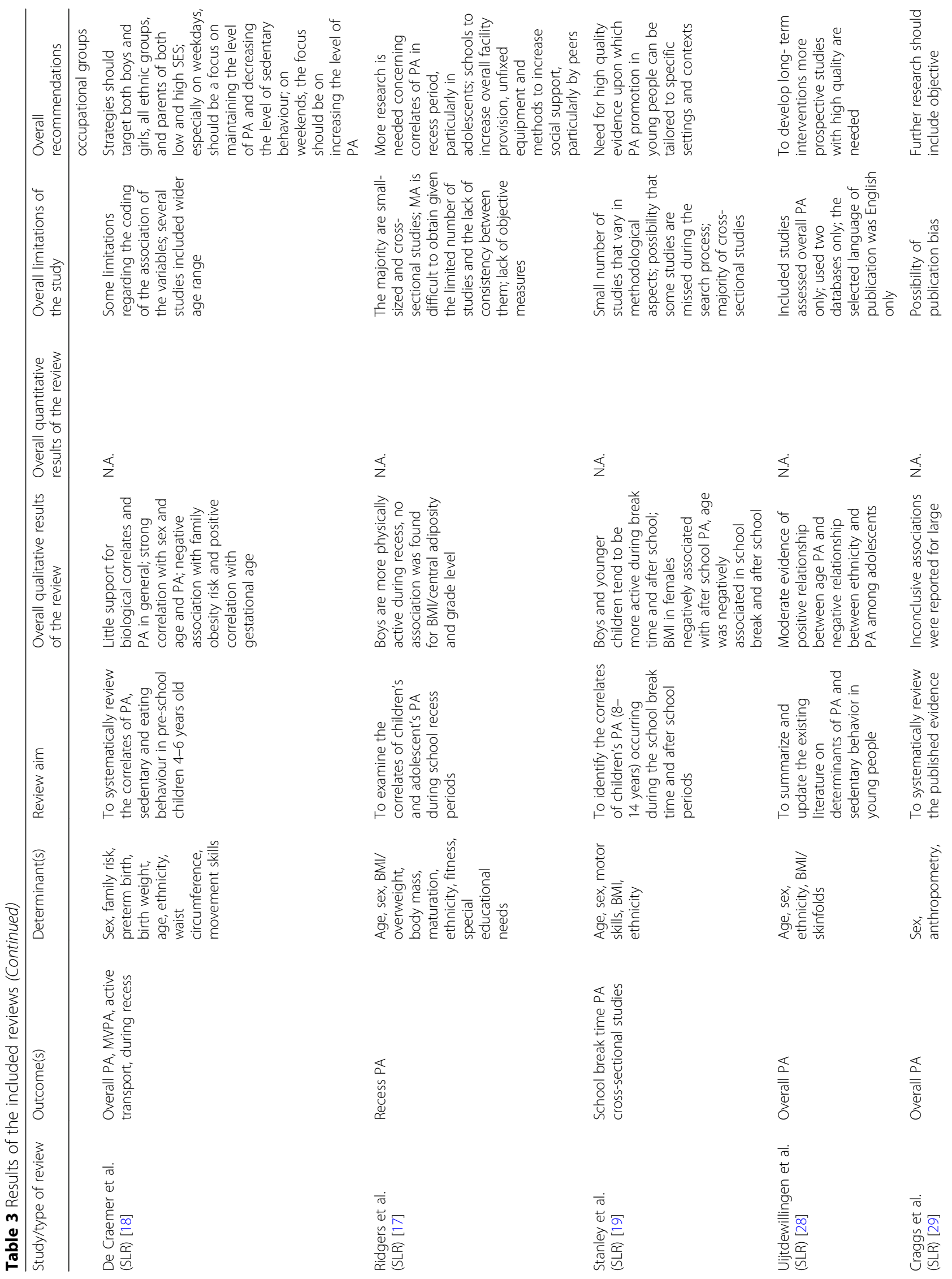




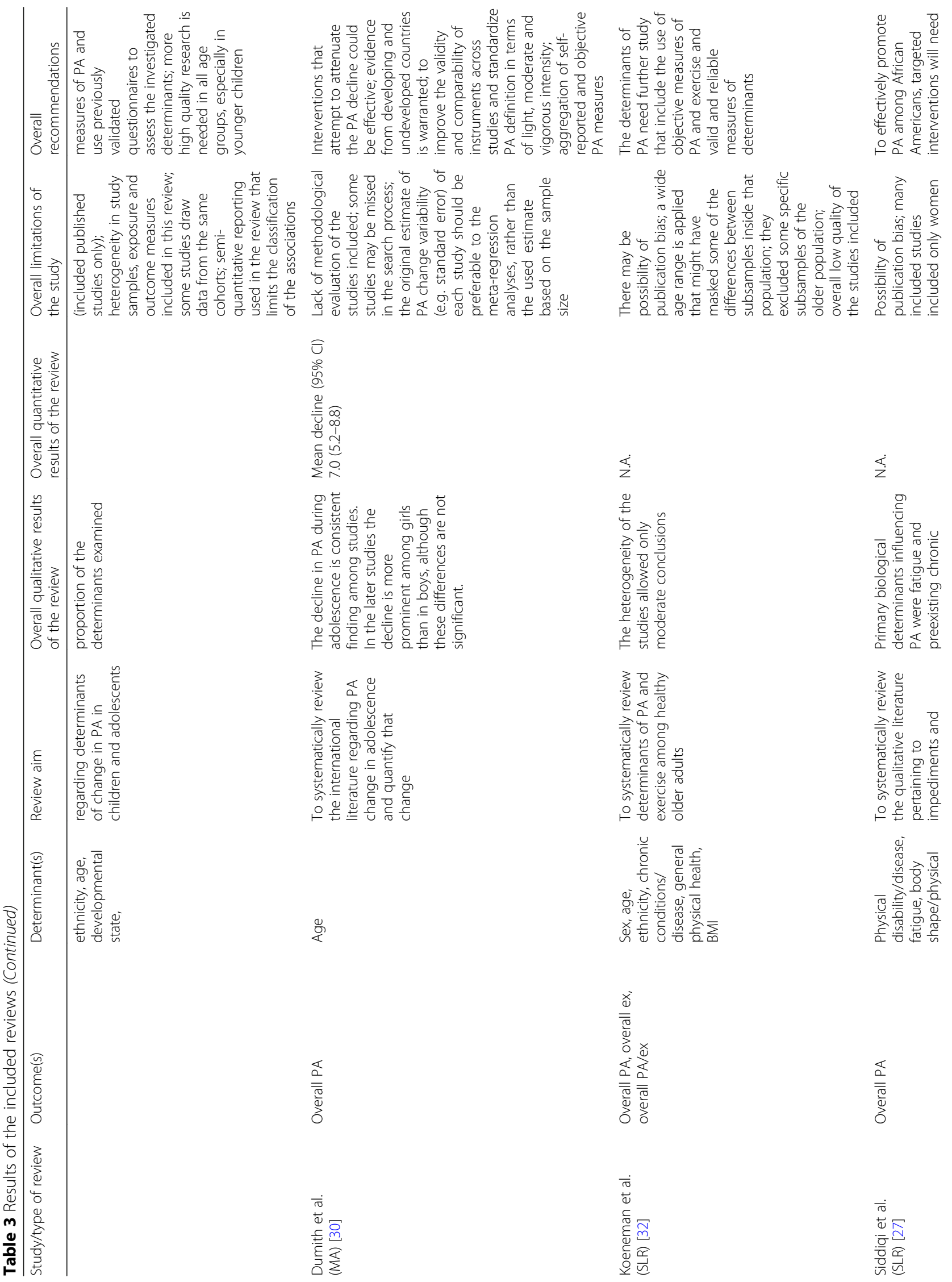




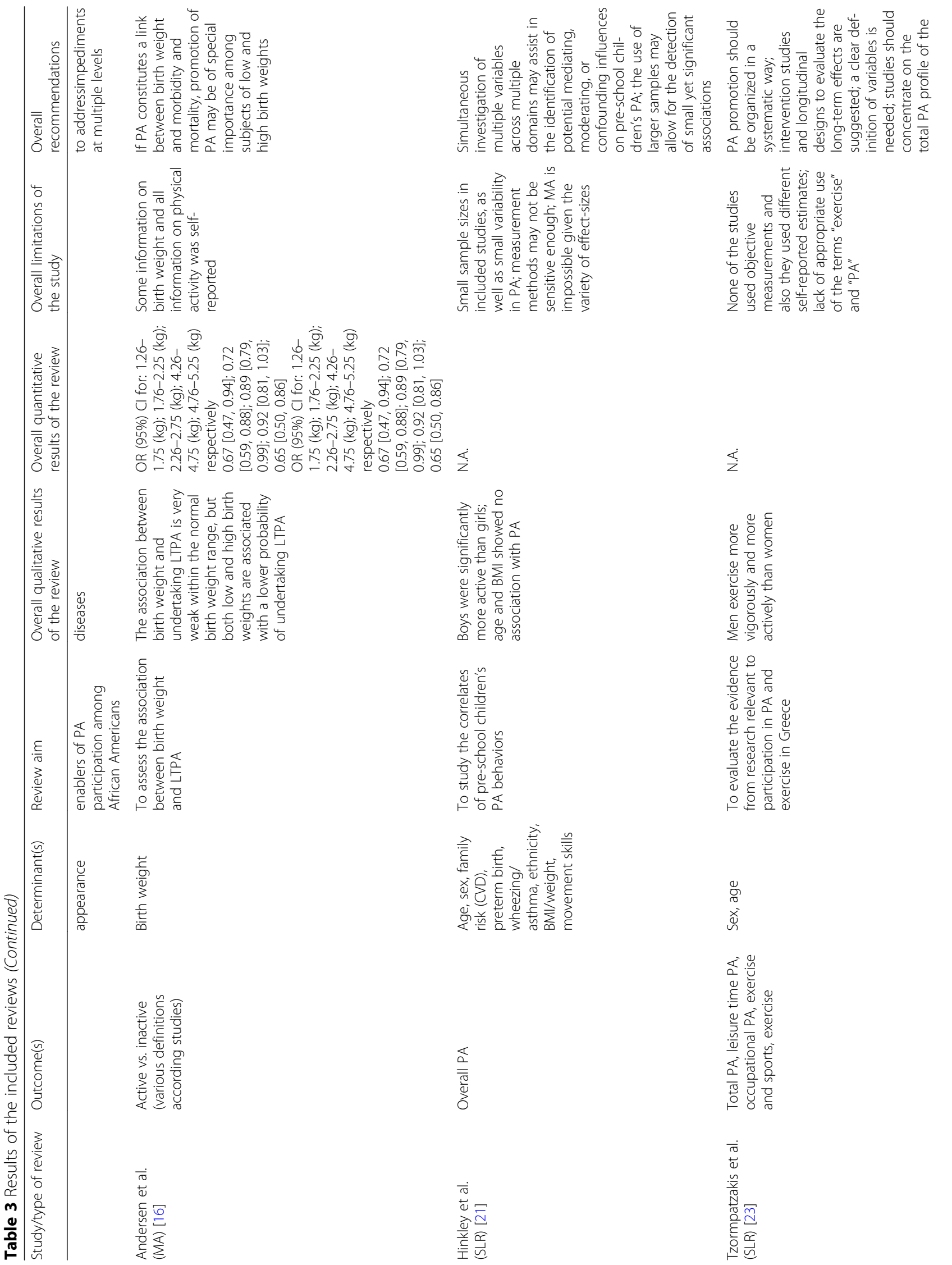




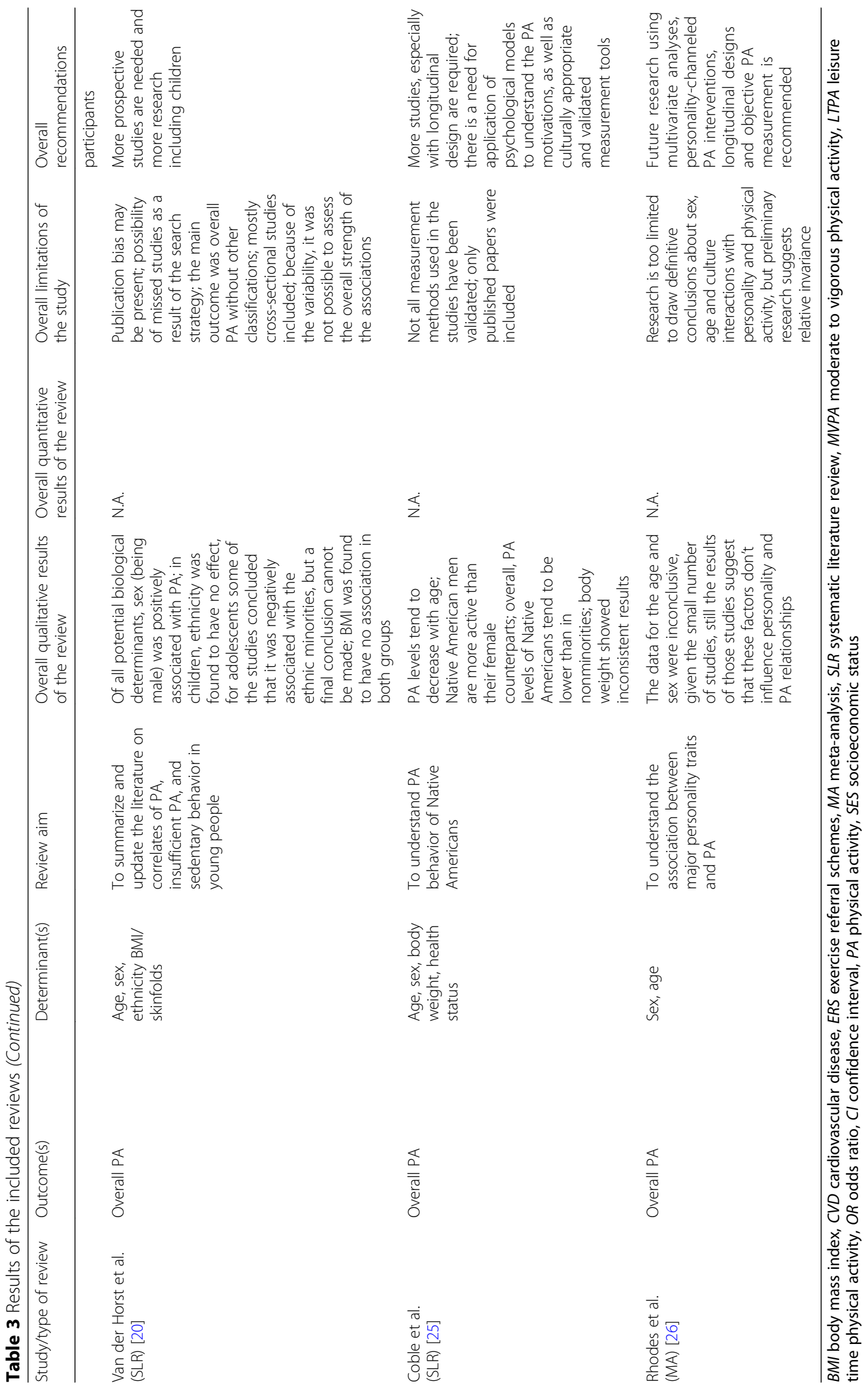


energy levels as determinants of PA [17-19, 21, 22, 27]. Furthermore, birth weight was studied in three reviews $[16,18,31]$, and anthropometry or body shape/waist circumference in two $[18,29]$. Finally, two reviews included maturation/level of development in adolescents [17, 29], one special educational needs as determinants of PA [17], and one included early growth and motor development [31].

\section{Measurement Methods of PA}

The majority of the eligible original studies used non-objective measurement methods of PA assessment (e.g., self-reporting, attendance reports) [16, 17, 19-33]. Objective measurements of PA, assessed by either accelerometer or pedometer, were used in 87 of the eligible original studies, included in 9 of the included reviews $[17,19-21,24,28-30,32]$. One review did not report the exact number of the studies that used objective and non-objective measures [18].

\section{Evaluation of the Quality of the SLRs and MAs}

The results of the quality assessment are reported in Table 4. Among the 19 included reviews, 13 were of moderate quality, 2 reviews were evaluated as weak [20, $23]$, and 4 as strong [15, 30, 31, 34]. From those reviews that were of moderate quality, nine $[17-19,21,22,25$, $26,30,33]$ were scored with four points and four [24, 27-29] received a quality rating of either six or seven. The characteristics of the included studies were provided by the majority of the reviews (16 out of 19 reviews); however, only 5 out of 19 reviews provided the list of the included and excluded studies. Furthermore, only 4 out of 19 reviews used the status of publication as an inclusion criterion and 2 out of 19 assessed the probability of publication bias.

\section{Summary of the Results of the Included Reviews by Importance of Determinants and Strength of Evidence}

Table 5 summarizes the results of the associations between the investigated biological determinants and PA, stratified in different age groups.

\section{Pre-School and Older Children}

Among pre-school children and older children, for most of the determinants, the reviews reported mixed findings (0, (importance of determinant), Ls (strength of evidence), Table 5). However, among pre-school children, family risk, preterm birth $[18,21]$, and low health status [21] were negatively correlated to overall PA and/or reported an effect size larger than 0.30 in more than $75 \%$ of the identified reviews assessing these two categories of determinants (+, Ls, Table 5). The results were based on studies that were mainly cross-sectional in design. Similarly, being female [18] and lower physical fitness levels [18] are negatively related to moderate vigorous PA (MVPA) among pre-school children. These findings are based on studies of both cross-sectional and cohort study design showing fairly consistent associations $(+, \mathrm{Pe}$, Table 5). BMI [21], birth weight [18] based on probable evidence (coded as (-, Pe) in Table 5), and anthropometry/body shape [18], based on limited, suggestive evidence (-, Ls, Table 5) were found to have no association with overall PA in pre-school children.

\section{Adolescents}

In the adolescents group, increasing age and females [20, 28-30] were found to be negatively associated with PA. Because of the mixed and contradictory results in part of the studies, these associations are probable $(+, \mathrm{Pe}$, Table 5). No association between body shape and PA among adolescents (-, Pe, Table 5) was found in one review [29].

\section{Children and Adolescents}

Among the reviews that included children and adolescents together $[17,20,28,29,34]$, age was found to be associated with PA (0, Ls), while sex was associated with PA (+, Ls). Birth weight $[18,31]$ was found not to be associated with PA with convincing strength of evidence $(--$, Ce) (Table 5).

\section{Adults}

Rural women were a particular adult category investigated by one review only [22]. It emerged that among these women, increasing age and BMI with limited, suggestive levels of evidence (+, Ls, and ++ , Ls, Table 5), and lower health status and physical fitness levels with a probable level of evidence $(++, \mathrm{Pe}$, and + , Pe, Table 5) respectively are negatively associated to PA.

When adults aged over 18 years were considered together, normal birth was found with convincing strength of evidence to be positively associated to PA and/or reported a significant effect size larger than 0.30 in all identified eligible studies included in the sole review assessing this particular category [16] (+, Ce, Table 5). Additionally, younger age [22, 23, 25], Caucasian ethnicity [24], better health status [22, 27, 35], and higher physical fitness levels [22, 27] were again found to be consistently positively associated to PA with a probable level of evidence among adults over 18 years of age $(++$, $\mathrm{Pe}$, Table 5), and males [23-26] were found to be positively associated to PA in more than $75 \%$ of the included studies in the reviews $(+, \mathrm{Pe}$, Table 5$)$.

\section{Discussion}

The aim of this umbrella systematic review was to summarize the evidence that has been produced to date about the biological determinants of PA across the life 


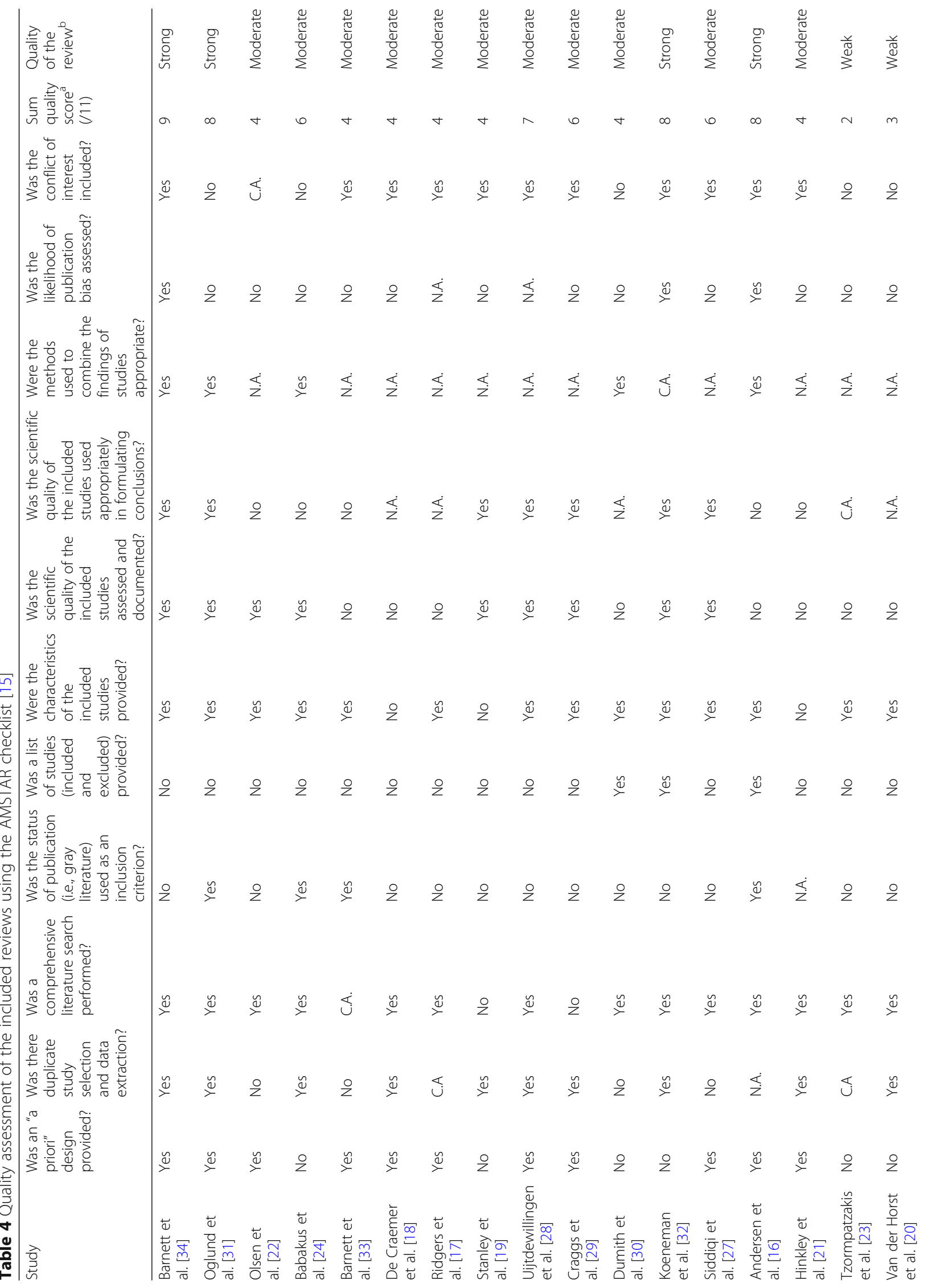




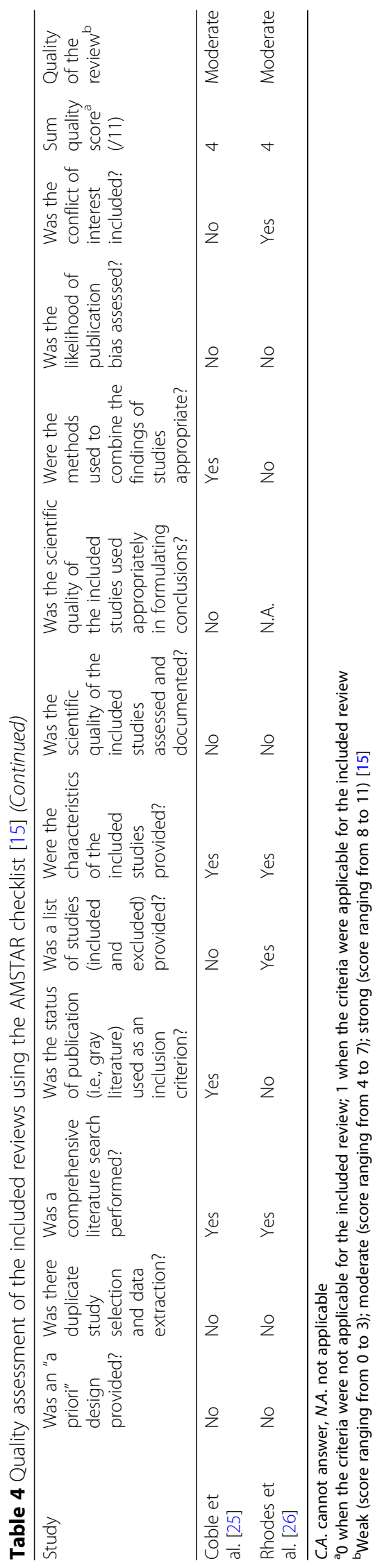




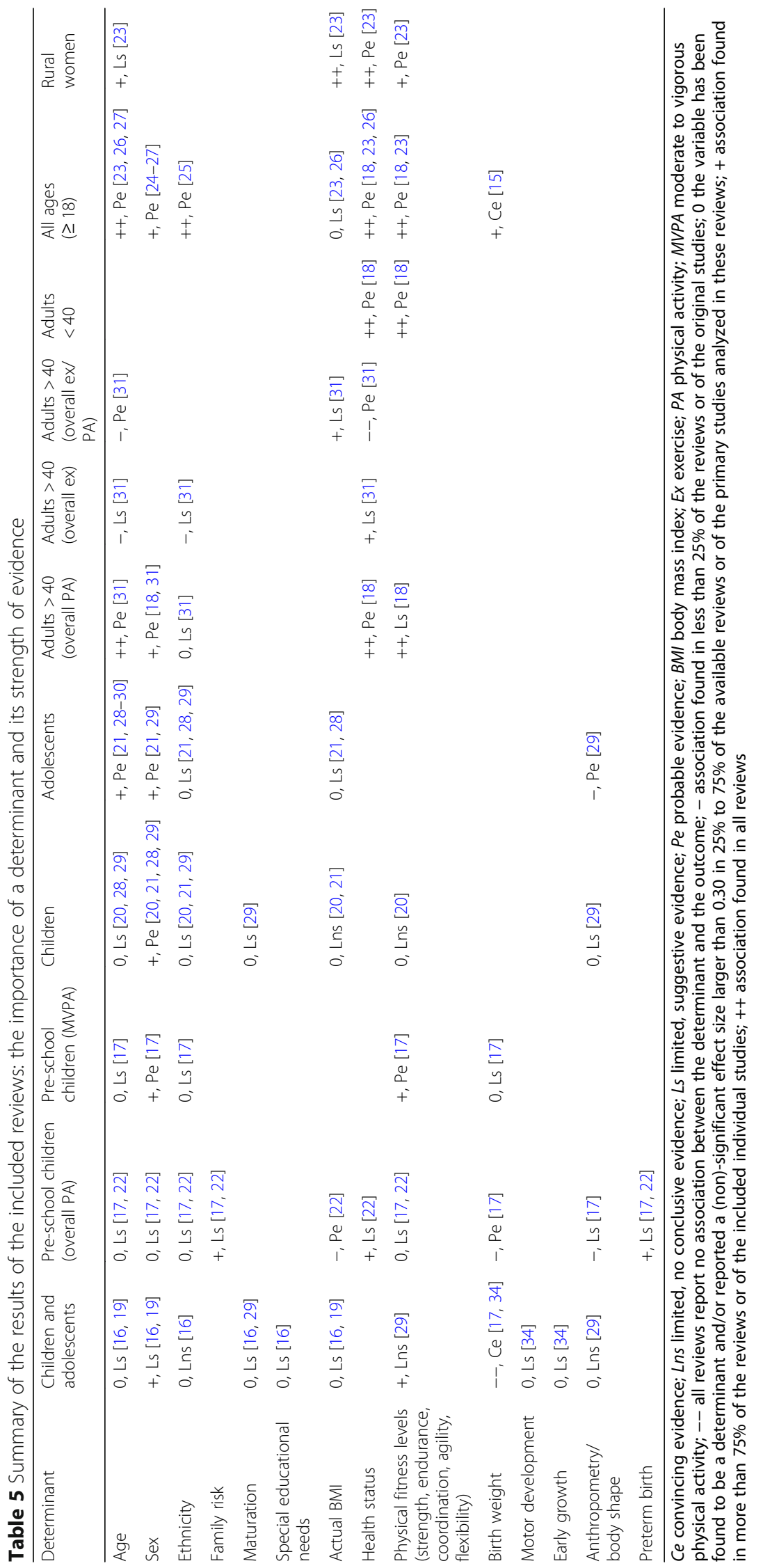


course. For most of the determinants, the strength of the level of evidence of the association with PA was mixed or probable. Few of the investigated determinants had convincing strength of evidence (Ce), either because of the lack of consistency of the results between the included studies or because of the small number of cohort studies investigating the specific determinants.

Determinants that were studied most frequently among all ages and demonstrated evidence suggesting a positive association to PA were younger age, being male, higher health status, and higher physical fitness levels.

Being female was negatively associated to PA participation in children, adolescents, and adults. The included reviews suggest that starting from adolescence and later, in adult life, increasing age is negatively associated to PA. Many reasons may explain these trends and greater understanding of the influence of additional contextual factors is required for both the sex and age determinants.

Apart from a biological background that could explain the avoidance of PA among older adults because of reduced physical capacity for everyday activities [32], other factors that change with age, such as social or behavioral, family, work status, or lifestyle, may have influence at different periods of life [36]. The observed sex difference in PA participation also may have a socio-cultural background. It is hypothesized that in women and adolescent girls, discouraging family/social environments could determine the observed sex-related differences in PA participation $[37,38]$. Our findings are in line with the most recent survey on PA in the citizens of the European Union [36], which indicates steady decrease in PA participation advancing after 24 years of age and lower PA levels in females.

Among pre-school children and older children, the results were mixed, with exception of the negative association between being female and MVPA among preschool children. The reasons behind these mixed results, as reported by the reviews, are small sample sizes, high diversity of the population included between studies, and the diversity of the measurement methods of PA used among the primary studies [18, 21].

Lower physical fitness levels and health status among adults were consistently found to be negatively associated to PA and reported as barriers to participation in PA [22, 25, 27, 32]. In contrary, PA is considered to have an important role in maintaining and improving the health status [39] indicating that special programmes targeting this particular group could be beneficial.

Normal birth weight was the only determinant for which there was convincing strength of evidence of positive association with PA among adults. This evidence is based on one MA of cohort studies that included adolescents and adults [16]. However, these results should be interpreted with caution because the quality of the individual studies included in this MA was not assessed and it included only population from the Nordic countries in Europe. Contrary to this review, two other MA and SLR that investigated the association of birth weight and PA among children and adolescents $[18,31]$ found no association. Although Andersen et al. [16] included adolescents in their study, they did not analyze the data in a way to assess the association specifically for this age group. However, the age stratification between younger and older than 35 years showed lower association between birth weight and PA in the younger participants' group [16]. According to the above-mentioned reviews $[16,18,31]$, normal birth weight was positively associated to PA only among adults. It is proposed that the rapid infant growth among those with lower birth weight may lead to adiposity later in life, which has negative impact on PA [31]. Based on these three reviews, it can be proposed that the normal birth weight might be positively correlated to PA among adults only.

Ethnicity was commonly studied as a determinant but, except for the adults $>18$ years, the results were usually mixed or insufficient to make final conclusions. The investigated ethnic groups differ among studies and reviews, which may contribute to the inconsistency of results. Also, since many reviews compared immigrants and ethnic minorities with the general population of the countries $[17-21,24,28,29,32,33,40]$, there is a possibility of bias by socioeconomic status that was not controlled in all of the individual studies.

BMI was another determinant with insufficient evidence among all age groups, due to mixed results among studies or lack of studies of longitudinal design that considered this determinant. A recent cohort study of older children showed that increased adiposity is associated to reduction of PA [41], but as yet no SLR/MA confirmed that.

Family risk for obesity and cardiovascular diseases was found to be negatively associated with PA among preschool children, but the strength of evidence is insufficient $[18,21]$. The same strength of evidence was found for most of the determinants investigated among children and adolescents, due to the large variation in the determinants investigated in different studies, which meant few could be compared, and the abundance of cross-sectional studies and lack of longitudinal investigations.

The majority of the studies included in the reviews were done in continents that include more developed countries. As a consequence, some determinants that may be characteristic and more relevant among less developed countries may not be shown.

Additionally, most of the included reviews were of moderate methodological quality. Most of them did not include gray literature and the probability of publication bias was rarely assessed. Additionally, half of 
the reviews did not assess the methodological quality of the studies and did not provide a list of excluded studies.

Additionally, PA was almost always assessed only in general terms (overall PA), rather than specific types of activity (e.g., leisure time, house activity, active travel) and was not defined clearly and uniformly among studies $[16,17,20,22-26,29,30,32-34]$. PA may have different patterns among sex, age, or socio-cultural contexts, which creates the possibility of bias when comparing the amount of PA between populations. Also, the lack of unified measurement methods of PA is an additional problem that was encountered among all the reviews. Specific definitions of PA may reveal greater insights into the determinants of PA behavior and together with a standardization of the assessment methods would enable a greater comparability among studies.

In addition, future studies on the mechanisms that underlie the proposed associations are needed in order to improve the knowledge about the biological determinants that influence PA.

\section{Conclusions}

Despite the limitations, there are still recommendations that can be drawn from this umbrella review. Age, sex, birth weight, health status, and physical fitness levels should be taken into consideration when introducing interventions aimed at increasing PA. Age, sex, and birth weight are non-modifiable factors, but special attention should be given to the possible social and behavioral interactions that may cause the observed associations. Creating programmes targeted at diverse ages, female population, and people with non-normal birth weight can be helpful. In addition, since poor health status and lower physical fitness levels were often found as a barrier to participating in PA, it is recommended to adopt separate interventions according to the individual's capacity for PA.

\section{Abbreviations \\ BMI: Body mass index; Ce: Convincing evidence; DEDIPAC: Determinants of Diet and Physical Activity; KH: Knowledge Hub; Lns: Limited, no conclusive evidence; Ls: Limited suggestive evidence; MAs: Meta-analyses; NCDs: Non- communicable diseases; PA: Physical activity; Pe: Probable evidence; SLRs: Systematic literature reviews; WHO: World Health Organization}

\section{Acknowledgments}

The authors thank Lien N, Lakerveld J, Mazzocchi M, O'Gorman D, Monsivais P, Nicolaou M, Renner B, Volkert D, and the DEDIPAC-HK Management team for their helpful support.

\section{Funding}

\footnotetext{
o 1, 19, 20 MIUR: CDR2.PRIN 2010/11 COD. 2010KL2Y73_003.

$\circ{ }^{1}$ Eraweb 2: contract n. 2013-2548/001-001-EMA2 for supporting the work of Katina Aleksovska.

$0^{2}$ Federal Ministry of Education and Research, Germany (01EA1377).

$0^{3,5,13,15}$ The Health Research Board, Ireland.

o ${ }^{8}$ MIUR: DEDIPAC F.S. 02.15 .02 COD. B84G14000040008

$\circ{ }^{11}$ Federal Ministry of Education and Research, Germany (01EA1374).
}

- ${ }^{14,17}$ This project was supported by grants from the Federal Ministry of Education and Research, Germany (Bundesministerium für Bildung und Forschung, Förderkennzeichen 01EA1372C and 01EA1372E. The responsibility for the content of this manuscript lies with the authors).

- ${ }^{18}$ Institut National de la Recherche Agronomique (INRA), Institut National de Prévention et d'Education pour la Sante (INPES).

Availability of Data and Materials

All data generated or analyzed during this study are included in this published article.

\section{Authors' Contributions}

Conceptualization: KA, AP, LG, CB, CB, GC, SC, DC, MC, GC, SD'H, MDC, ADB, SH, $L I, P I, \sqcup, M K, F L, A L, G N$, J-AN, TP, AP, AS, HS, AS, WS, WR, CMD, LC, SB. Data curation: KA, AP, LG, MC, GC, CC, FL, GN, J-AN, CP, CMD, LC, SB. Formal analysis: KA. Funding acquisition: GC, AS, WS, CMD, LC, SB. Investigation: CC. Methodology: KA, AP. Visualization: CB. Writing —original draft: KA, AP. Writing - review and editing: KA, AP, LG, CB, CB, GC, AC, DC, MC, GC, TC, CC, SD'H, MDC, ADB, SH, LI, J, $\mathrm{PI}, \sqcup, \mathrm{MK}, \mathrm{AK}, \mathrm{FL}, \mathrm{AL}, \mathrm{GN}, \mathrm{J}-\mathrm{AN}, \mathrm{CP}, \mathrm{TP}, \mathrm{AP}, \mathrm{AS}, \mathrm{HS}, \mathrm{RS}, \mathrm{AS}, \mathrm{WS}, \mathrm{WR}, \mathrm{CMD}, \mathrm{LC}, \mathrm{SB}$. All authors read and approved the final manuscript.

Ethics Approval and Consent to Participate Not applicable.

\section{Consent for Publication}

Not applicable.

\section{Competing Interests}

The authors, Katina Aleksovska, Anna Puggina, Luca Giraldi, Christoph Buck, Con Burns, Greet Cardon, Angela Carlin, Simon Chantal, Donatella Ciarapica, Marco Colotto, Giancarlo Condello, Tara Coppinger, Cristina Cortis, Sara D’Haese, Marieke De Craemer, Andrea Di Blasio, Sylvia Hansen, Licia lacoviello, Johann Issartel, Pascal Izzicupo, Lina Jaeschke, Martina Kanning, Aileen Kennedy, Fiona Ling, Agnes Luzak, Giorgio Napolitano, Julie-Anne Nazare, Camille Perchoux, Tobias Pischon, Angela Polito, Alessandra Sannella, Holger Schulz, Rhoda Sohun, Astrid Steinbrecher, Wolfgang Schlicht, Walter Ricciardi, Ciaran MacDonncha, Laura Capranica, and Stefania Boccia, declare that they have no competing interests.

\section{Publisher's Note}

Springer Nature remains neutral with regard to jurisdictional claims in published maps and institutional affiliations.

\section{Author details}

${ }^{1}$ Università Cattolica del Sacro Cuore, Sezione di Igiene, Istituto di Sanità Pubblica, Rome, Italy. ${ }^{2}$ Leibniz Institute for Prevention Research and Epidemiology-BIPS, Bremen, Germany. ${ }^{3}$ Department of Sport, Leisure and Childhood Studies, Cork Institute of Technology, Cork, Munster, Ireland. ${ }^{4}$ Department of Movement and Sports Sciences, Ghent University, Ghent, Belgium. ${ }^{5}$ Department of Physical Education and Sport Sciences, University of Limerick, Limerick, Ireland. ${ }^{6}$ Department of Applied Sciences in Physical Activity and Management, Catholic University of Valencia "San Vicente Mártir", Valencia, Spain. ${ }^{7}$ Council for Agricultural Research and Economics, Research Centre for Food and Nutrition, Rome, Italy. ${ }^{8}$ Department of Movement, Human and Health Sciences, University of Rome Foro Italico, Rome, Italy. ${ }^{9}$ Department of Human Sciences, Society, and Health, University of Cassino and Lazio Meridionale, Cassino, Italy. ${ }^{10}$ Department of Medicine and Aging Sciences, G. d'Annunzio' University of Chieti-Pescara, Chieti and Pescara, Italy. ${ }^{11}$ Department of Sport and Exercise Sciences, University of Stuttgart, Stuttgart, Germany. ${ }^{12}$ Department of Epidemiology and Prevention, IRCCS Istituto Neurologico Mediterraneo: NEUROMED, Pozzilli, Italy. ${ }^{13}$ School of Health and Human Performance, Multisensory Motor Learning Lab, Dublin City University, Dublin, Ireland. ${ }^{14}$ Molecular Epidemiology Group, Max Delbruck Center for Molecular Medicine in the Helmholtz Association (MDC), Berlin, Germany. ${ }^{15}$ Centre for Preventive Medicine, School of Health and Human Performance, Dublin City University, Dublin, Ireland. ${ }^{16}$ Institute of Sport, Exercise \& Active Living, Victoria University, Melbourne, Australia. ${ }^{17}$ Institute of Epidemiology I, Helmholtz Zentrum München, German Research Center for Environmental Health, Neuherberg, Germany. ${ }^{18} \mathrm{Centre}$ de Recherche en Nutrition Humaine Rhône-Alpes, CarMeN INSERM U1060, University of Lyon1, Lyon, France. ${ }^{19}$ Italian National Institute of Health, 
(Istituto Superiore di Sanita - ISS), Rome, Italy. ${ }^{20}$ Fondazione Policlinico Universitario A.Gemelli IRCCS, UOC Igiene Ospedaliera, Roma, Italia.

\section{Received: 23 August 2018 Accepted: 30 November 2018 Published online: 08 January 2019}

\section{References}

1. World Health Organization. Global recommendations on physical activity for health. Geneva: World Health Organization; 2010. p. 60

2. World Health Organization. Health statistics 2014 [Internet]. 2014.

3. Reiner M, Niermann C, Jekauc D, Woll A. Long-term health benefits of physical activity - a systematic review of longitudinal studies. BMC Public Health. 2013;13:813.

4. Kindig DA. Understanding population health terminology. Milbank Q. 2007; 85(1):139-61. Available from: http://www.ncbi.nlm.nih.gov/pubmed/17319809

5. Bauman AE, Reis RS, Sallis JF, Wells JC, Loos RJF, Martin BW. Correlates of physical activity: why are some people physically active and others not? Lancet. 2012;380(9838):258-71.

6. Ooms L, Veenhof C, Schipper-van Veldhoven N, de Bakker DH. Sporting programs for inactive population groups: factors influencing implementation in the organized sports setting. BMC Sport Sci Med Rehabil. 2015;7:12.

7. Sallis JF, Cervero RB, Ascher W, Henderson KA, Kraft MK, Kerr J. An ecological approach to creating active living communities. Annu Rev Public Health. 2006;27:297-322.

8. Glass TA, McAtee MJ. Behavioral science at the crossroads in public health extending horizons, envisioning the future. Soc Sci Med. 2006;62(7):1650-71.

9. Sterdt E, Pape N, Kramer S, Liersch S, Urban M, Werning R, et al. Do children's health resources differ according to preschool physical activity programmes and parental behaviour? A mixed methods study. Int J Environ Res Public Health. 2014;11:2407-26.

10. Biddle SJH, Atkin AJ, Cavill N, Foster C. Correlates of physical activity in youth: a review of quantitative systematic reviews. Int Rev Sport Exerc Psychol. 2011;4(1):25-49. Available from: https://doi.org/10.1080/1750984X. 2010.548528

11. Bauman AE, Sallis JF, Dzewaltowski DA, Owen N. Toward a better understanding of the influences on physical activity: the role of determinants, correlates, causal variables, mediators, moderators, and confounders. Am J Prev Med. 2002;23(2 Suppl):5-14

12. Liberati A, Altman DG, Tetzlaff J, Mulrow C, Gotzsche PC, loannidis JPA, et al The PRISMA statement for reporting systematic reviews and meta-analyses of studies that evaluate healthcare interventions: explanation and elaboration. BMJ. 2009;339:b2700

13. Capranica L, Donncha CM, Puggina A. Determinants of physical activity : an umbrella systematic literature review, PROSPERO International prospective register of systematic reviews; 2015. p. 1-5.

14. Sleddens EFC, Kroeze W, Kohl LFM, Bolten LM, Velema E, Kaspers PJ, et al. Determinants of dietary behavior among youth: an umbrella review. Int J Behav Nutr Phys Act. 2015;12:7.

15. Shea BJ, Grimshaw JM, Wells GA, Boers M, Andersson N, Hamel C, et al. Development of AMSTAR: a measurement tool to assess the methodological quality of systematic reviews. BMC Med Res Methodol. 2007;7:10.

16. Andersen LG, Angquist L, Gamborg M, Byberg L, Bengtsson C, Canoy D, et al. Birth weight in relation to leisure time physical activity in adolescence and adulthood: meta-analysis of results from 13 Nordic cohorts. PLoS One. 2009;4(12):e8192

17. Ridgers ND, Salmon J, Parrish A-M, Stanley RM, Okely AD. Physical activity during school recess: a systematic review. Am J Prev Med. 2012;43(3):320-8.

18. De Craemer M, De Decker E, De Bourdeaudhuij I, Vereecken C, Deforche B, Manios $Y$, et al. Correlates of energy balance-related behaviours in preschool children: a systematic review. Obes Rev. 2012;13(Suppl 1):13-28.

19. Stanley RM, Ridley K, Dollman J. Correlates of children's time-specific physical activity: a review of the literature. Int J Behav Nutr Phys Act. 2012; 9(1):50. Available from: https://doi.org/10.1186/1479-5868-9-50

20. Van Der Horst K, Paw MJCA, Twisk JWR, Van Mechelen W. A brief review on correlates of physical activity and sedentariness in youth. Med Sci Sports Exerc. 2007;39(8):1241-50.

21. Hinkley T, Crawford D, Salmon J, Okely AD, Hesketh K. Preschool children and physical activity. Am J Prev Med. 2008;34(5):435-41. e7
22. Olsen JM. An integrative review of literature on the determinants of physical activity among rural women. Public Health Nurs. 2013;30(4): 288-311.

23. Tzormpatzakis N, Sleap M. Participation in physical activity and exercise in Greece: a systematic literature review. Int J Public Health. 2007;52(6):360-71.

24. Babakus WS, Thompson JL. Physical activity among South Asian women: a systematic, mixed-methods review. Int J Behav Nutr Phys Act. 2012;9:150.

25. Coble JD, Rhodes RE. Physical activity and native Americans: a review. Am J Prev Med. 2006;31(1):36-46.

26. Rhodes RE, Smith NEl. Personality correlates of physical activity: a review and meta-analysis. Br J Sports Med. 2006:40:958-65.

27. Siddiqi Z, Tiro JA, Shuval K. Understanding impediments and enablers to physical activity among African American adults: a systematic review of qualitative studies. Health Educ Res. 2011;26(6):1010-24.

28. Uijtdewilligen L, Nauta J, Singh AS, van Mechelen W, Twisk JWR, van der Horst $\mathrm{K}$, et al. Determinants of physical activity and sedentary behaviour in young people: a review and quality synthesis of prospective studies. $\mathrm{Br}$ J Sports Med. 2011;45(11):896-905.

29. Craggs C, Corder K, van Sluijs EMF, Griffin SJ. Determinants of change in physical activity in children and adolescents: a systematic review. Am J Prev Med. 2011;40(6):645-58.

30. Dumith SC, Gigante DP, Domingues MR, Kohl HW. Physical activity change during adolescence: a systematic review and a pooled analysis. Int $J$ Epidemiol. 2011:40(3):685-98.

31. Oglund GP, Hildebrand M, Ekelund U. Are birth weight, early growth, and motor development determinants of physical activity in children and youth? A systematic review and meta-analysis. Pediatr Exerc Sci. 2015;27(4): 441-53.

32. Koeneman MA, Verheijden MW, Chinapaw MJM, Hopman-Rock M. Determinants of physical activity and exercise in healthy older adults: a systematic review. Int J Behav Nutr Phys Act. 2011;8:142.

33. Barnett I, Guell C, Ogilvie D. The experience of physical activity and the transition to retirement: a systematic review and integrative synthesis of qualitative and quantitative evidence. Int J Behav Nutr Phys Act. 2012;9(1): 97. Available from: https://doi.org/10.1186/1479-5868-9-97

34. Barnett LM, Lai SK, Veldman SLC, Hardy LL, Cliff DP, Morgan PJ, et al. Correlates of gross motor competence in children and adolescents: a systematic review and meta-analysis. Sports Med. 2016;46(11):1663-88

35. Cobley S, Baker J, Wattie N, McKenna J. Annual age-grouping and athlete development: a meta-analytical review of relative age effects in sport. Sports Med. 2009;39(3):235-56

36. EU. Special Eurobarometer 412 "Sport and physical activity". Special Eurobarometer. 2014:412:1-135.

37. Bailey R, Wellard I, Dismore H. Girls' participation in physical activities and sports: benefits, patterns, influences and ways forward. Benefits Phys Act Tech Pap WHO 2004;1-30. Available from: https://www.icsspe.org/sites/ default/files/Girls.pdf

38. El Ansari W, Lovell G. Barriers to exercise in younger and older nonexercising adult women: a cross sectional study in London, United Kingdom. Int J Environ Res Public Health. 2009;6:1443-55.

39. Haskell WL, Lee I-M, Pate RR, Powell KE, Blair SN, Franklin BA, et al. Physical activity and public health: updated recommendation for adults from the American College of Sports Medicine and the American Heart Association. Med Sci Sports Exerc. 2007;39(8):1423-34

40. Pavey T, Taylor A, Hillsdon M, Fox K, Campbell J, Foster C, et al. Levels and predictors of exercise referral scheme uptake and adherence: a systematic review. J Epidemiol Community Health. 2012;66(8):737-44.

41. Richmond RC, Davey Smith G, Ness AR, den Hoed M, McMahon G, Timpson NJ. Assessing causality in the association between child adiposity and physical activity levels: a Mendelian randomization analysis. PLoS Med. 2014; 11(3):e1001618 